This is a postprint version of the following published document:

Merino, M., Ahedo, E. Contactless steering of a plasma jet with a 3D magnetic nozzle, In: Plasma sources science and technology, 26, 095001, Aug. 2017, 10 Pp.

DOI: https://doi.org/10.1088/1361-6595/aa8061

(C) 2017 IOP Publishing Ltd. 


\title{
Contactless steering of a plasma jet with a 3D magnetic nozzle
}

\author{
Mario Merino, a) and Eduardo Ahedo ${ }^{1}$ \\ Equipo de Propulsión Espacial y Plasmas (EP2), Universidad Carlos III de Madrid, 28911 Leganés, Spain
}

A 3D, steerable magnetic nozzle is presented that enables contactless thrust vector control of a plasma jet without any moving parts. The concept represents a substantial simplification over current plasma thruster gimbaled platforms, and requires only a small modification in thrusters that already have a magnetic nozzle. The characteristics of the plasma expansion in the 3D magnetic field and the deflection performance of the device are characterized with a fully-magnetized plasma model, suggesting that thrust deflections of 5-10 deg are readily achievable.

PACS numbers: 52.75.Di, 52.30.Ex, 52.59.Dk

Keywords: electric space propulsion, thrust vector control, magnetic nozzles

\section{INTRODUCTION}

All spacecraft using plasma thrusters need a thrust vector control (TVC) device to modify the direction of the thrust force during the mission. Deflections of about 8-10 deg in all directions are sufficient to compensate center of mass displacements and to cover the requirements of the vast majority of current propulsive missions ${ }^{1,2}$. Present TVC solutions consist on mounting the thrusters on complex and heavy gimbaled platforms ${ }^{3,4}$ or robotic $\operatorname{arms}^{5}$. As with any other moving part, they are a costly and delicate piece of equipment that may affect the overall reliability of the system. Moreover, these pointing mechanisms introduce a number of additional issues, such as the need for flexible piping and connectors to the thruster, higher complexity for thermal control, and the damping of shocks and vibrations. TVC concepts using mechanically-displaced ion grid optics ${ }^{6}$, asymmetric gas injection and magnetic fields in Hall effect thrusters ${ }^{7}$, or acting on the plasma jet by means of a fixed, large external coil located on one flank of the thruster ${ }^{8}$ have been proposed as alternatives.

Magnetic nozzles ${ }^{9,10}$ (MNs) serve as the contactless acceleration stage of several next-generation plasma thrusters, including the Helicon Plasma Thruster ${ }^{11,12}$ (HPT), the Electron-Cyclotron-Resonance thruster ${ }^{13,14}$ (ECRT) the Applied-field magneto-plasma-dynamic thruster $^{15,16}$ (AF-MPDT) and the VAriable Specific Impulse Magneto-Rocket (VASIMR) ${ }^{17-19}$. Other thrusters like the High-Efficiency Multistage Plasma Thruster $(\text { HEMPT })^{20}$ and the Diverging Cusped Field Thruster $(\mathrm{DCFT})^{21}$ have MN-like magnetic configurations. In their basic design, MNs consist of an axisymmetric, convergent-divergent magnetic field $\boldsymbol{B}$ that guides the expansion of a hot plasma to form a supersonic jet. Their operation principles are now well understood ${ }^{22-26}$ and have been observed experimentally ${ }^{18,19,27-33}$ : in the MN, ions gain axial kinetic energy at the expense of electron internal energy thanks to the mediation of the selfconsistent electric field. Additional plasma acceleration

\footnotetext{
a) Electronic mail: mario.merino@uc3m.es; http://ep2.uc3m.es
}

mechanisms exist in some devices like the VASIMR which rely on ion internal energy rather than electron internal energy. Correct operation of the $\mathrm{MN}$ requires that at least the electrons be well magnetized; the reaction to the magnetic forces that shape the plasma expansion are felt on the magnetic circuit of the thruster, creating magnetic thrust. Finally, the plasma detaches from the guiding magnetic field downstream due to demagnetization and inertia.

Typical laboratory prototypes of these technologies run at powers from $100 \mathrm{~W}$ to $30 \mathrm{~kW}$, and on propellants that go from light ones, as hydrogen, to heavy ones, as xenon. The diameter of the plasma source ranges from 1-10 cm, and magnetic strengths of $0.04-4 \mathrm{~T}$ are used. To provide two specific examples of actual test devices relevant to the present study, the VASIMR (in helicon source-only mode) has been operated on argon at 30 $\mathrm{kW}$ with magnetic strengths up to $0.17-2 \mathrm{~T}$, reaching near-full ionization, plasma densities and electron temperatures around $10^{19}-10^{20} \mathrm{~m}^{-3}$ and $10 \mathrm{eV}$ in the exhaust plume ${ }^{18,19}$; the ECRT at ONERA operates on a range of gases at $0.1 \mathrm{~T}$, densities $10^{18}-10^{19} \mathrm{~m}^{-3}$, and electron temperatures of $10-25 \mathrm{eV}^{14}$. Except in devices where direct ion heating is applied (e.g. VASIMR with ICRH stage), the ion temperature is a small fraction of the electron temperature. The plasma in the thruster plume is near-collisionless, with mean-free paths that increase rapidly downstream as the plasma expands and the neutral and plasma densities decrease, and values at the MN throat already larger than the characteristic radius of the plasma (about a factor 4 in the VASIMR in helicon source-only mode ${ }^{19}$; about $10^{4}$ for the ECRT at ONERA $^{14}$ ).

A non-symmetric MN configuration would allow not only to guide and expand the plasma jet, but also to deflect it laterally in any direction to control the thrust vector. Following this line of thought, this work proposes and explores the theoretical performance of a novel TVC system for electric space propulsion based on a steerable or vectorial magnetic nozzle (VMN) that has no moving parts and can provide a significant simplification of the electric propulsion subsystem with respect to the current state of the art. The concept is particularly interesting for plasma thrusters that already incorporate an axisym- 
metric MN, as only a minor modification to the construction of the magnetic generator assembly is necessary to turn it into a VMN. The concept is likewise applicable to other plasma technologies such as plasma material treatments to control the region of deposition/erosion ${ }^{34,35}$.

The rest of the paper is structured as follows. Section II describes the construction, advantages and disadvantages of VMNs. The magnetic setup used in the analyses of the following sections is introduced there. Section III presents the 3D version of the the fullymagnetized plasma expansion model of Ref. ${ }^{36}$, which is then used in Section IV to discuss in detail the 3D plasma plume properties of the VMN. In Section V several simulations are used to analyze the TVC capabilities of the VMN. Finally, Section VI comments on the range of validity of the model and its results when a partiallymagnetized plasma is considered, and Section VII gathers the conclusions of this work.

\section{THE 3D VECTORIAL MAGNETIC NOZZLE}

A 3D VMN can be realized in several ways. A first, naïve approach relies on a gimbaled magnetic coil or permanent magnet array, which would be reoriented mechanically to steer the applied magnetic field in the required direction. This approach, however, does not offer any advantages in terms of simplicity and reliability with respect to other gimbaled TVC systems.

A second, more advantageous approach is to have an arrangement of $N \geq 3$ intertwined magnetic coils, whose position is fixed with respect to the thruster and whose electric current can be modified independently ${ }^{37,38}$. In this approach, each coil is tilted at an angle $\alpha$ with respect to the axis of the thruster, and precessed an angle $2 \pi / N$ with respect to each other to create a rotationalsymmetric configuration (Fig. 1). This way, no moving parts are required. Physically, this type of VMN may be constructed by winding simultaneously the $N$ coils on a circular spool, resulting in a set of interwoven, slightlyelliptic coils (Fig. 1 top), or by interlocking $N$ rigid circular coils of slightly different radii and/or slightly offset from the axis into that position (Fig. 1 bottom). By controlling the ampere-turns on each coil, it is possible to create an orientable 3D magnetic field according to the following principles:

1. When all coils carry an identical number of ampereturns, the system generates a MN whose axis coincides with the axis of the thruster (i.e., no deflection). The magnetic field in this case is nearaxisymmetric, with small asymmetries becoming important only at large distances from the axis (i.e., in the periphery of the nozzle, close to the coils). A larger angle $\alpha$ and a lower $N$ increase this asymmetry.

2. By choosing different ampere-turn values for each coil it is possible to break the symmetry and reorient the axis of the MN:

(a) If all the electric currents on the coils have the same sign, the axis of the MN can be oriented in any direction within a reference $N$ polygonal angular space, where the maximum deflection angle (at the vertices of this polygon) is $\alpha$. This polygon is depicted in Fig. 1 for $N=3,5$ and $\alpha=15 \mathrm{deg}$.

(b) By inverting the sign of some of the electric currents on the coils it is possible to generate deflections beyond that $N$-polygonal space, at the cost of a higher total electric current for the same field strength at the origin, and a
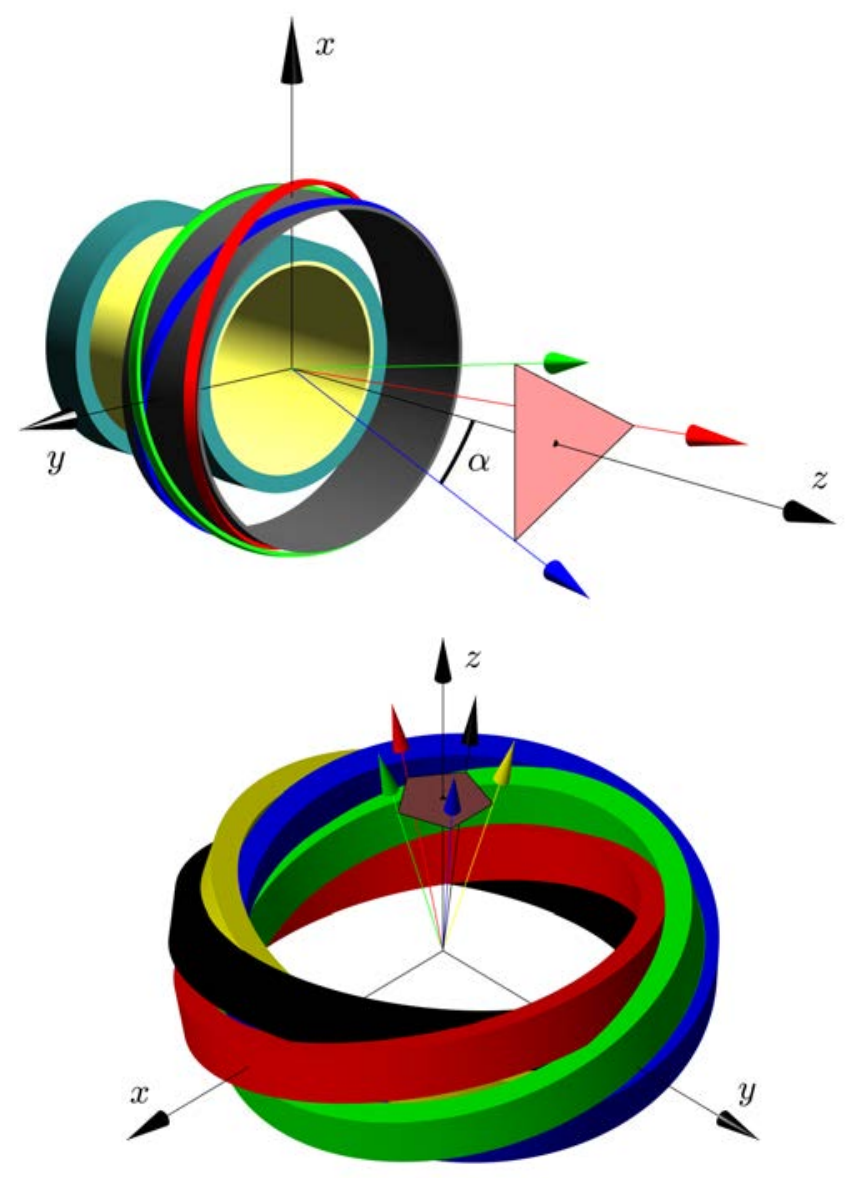

FIG. 1. Top: Sketch of a VMN consisting of $N=3$ elliptical coils (red, green blue) with $\alpha=15 \mathrm{deg}$, wound over a cylindrical spool and placed at the exit section of a HPT-like plasma source. The director vectors for each coil axis are displayed as colored arrows. The reference polygonal angular space in this case is a triangle (in pink). Bottom: VMN configuration built from $N=5$ interlocked circular coils with $\alpha=15 \mathrm{deg}$, whose center has been slightly offset from the origin of coordinates (plasma source not shown). The reference pentagon angular space is shown (in pink). 
higher asymmetry in the MN periphery.

In order to deflect the plasma jet without affecting the internal plasma dynamics and the internal efficiency of the device, it is desirable that the VMN control the shape and intensity of the magnetic field in the plume region without substantially modifying the internal magnetic topology of the plasma source. For plasma thrusters that have an essentially-axial applied magnetic field in the plasma source, this is achieved by placing the VMN arrangement at or near the exit plane of the plasma thruster. This way, the rest of the thruster magnetic circuit (e.g. a solenoid-like system or permanent magnets) exerts the dominant contribution to the internal magnetic field configuration. Naturally, increasing the relative strength of the VMN field and the deflection angle increases the influence of the VMN on the internal magnetic topology of the source.

To illustrate the tilted-coil VMN construction and its installation on a plasma thruster, consider a HPT- or ECRT-like plasma source of radius $R$ inside a solenoid of radius $R_{S}=2.5 R$ and length $L_{S}=8 R$, and a $N=3$ VMN of radius $R_{L}=6 R$ and $\alpha=15$ deg located at the exit of the source. The center of each VMN coil coincides with the origin of coordinates, and the director vector of the first coil is contained in the $y z$ plane. This magnetic circuit is studied in next Sections under several electric current configurations, described by the ratio of ampereturns between the source solenoid and each of the three VMN coils, in the form (solenoid):(coil 1):(coil 2):(coil 3), as gathered in Table I. Configuration $O$ creates a symmetric MN for reference with no deflection. Configuration $A$ and its variants steer the magnetic centerline toward one of the vertices of the reference triangle of Fig. 1. Configuration $B$ and its variants move it toward one triangle edge. Primed simulations have a net current in the coils 5 times stronger than unprimed ones, but keep the same solenoid current, resulting in a larger deflection angle and lower plume divergence rate. Simulations with a bar symbol have negative currents in one or two of the coils, tripling the sum of absolute values of the currents while keeping the same net current in the VMN for fair comparison. Figure 2 shows the magnetic circuit and the magnetic field in configuration $A$. Observe that the internal magnetic field remains almost unaffected by the VMN in this case.

\section{FULLY MAGNETIZED PLASMA MODEL}

To study the operation and performance of the VMN, the two-fluid plasma model of Ref. ${ }^{36}$ is adapted here to $3 \mathrm{D}$ magnetic configurations. This model is the fullymagnetized limit of the DIMAGNO model introduced in Ref. $^{24}$, and it is representative of the plasma expansion in the near-region plume in a HPT and similar devices at high magnetic field strength and/or light propellants.

The plasma is assumed to be composed of single-

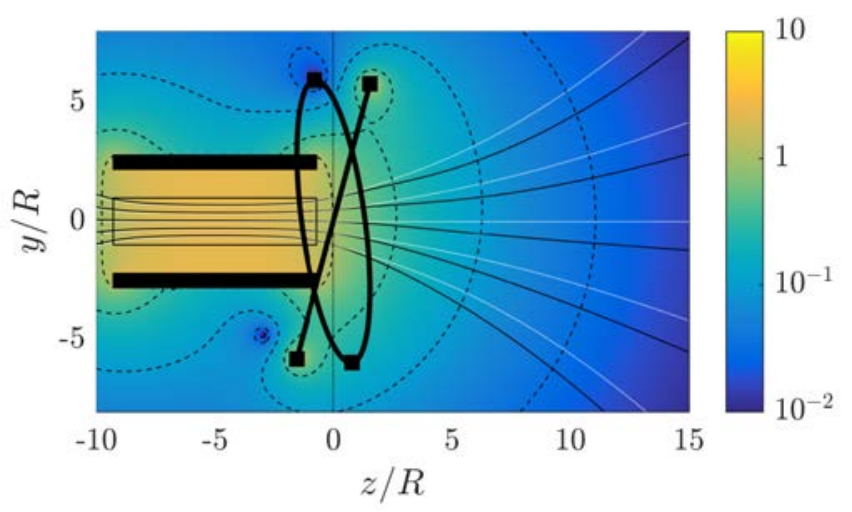

FIG. 2. Magnetic field generator example used in this work, composed of a solenoid of 15 circular current loops of radius $R_{S}=2.5 R$, extending from $z=-9 R$ to $z=-R$, and a VMN made of $N=3$ circular coils with $R_{L}=6 R, \alpha=15 \mathrm{deg}$ located at the $z=0$ plane. The black rectangle suggests the position of the plasma source, of radius $R$. The color map shows the magnetic field strength in the $x=0$ plane normalized with its value at the origin, $B / B_{0}$, when the ampereturn ratio between the solenoid and each of the three coils is $15: 1: 0: 0$ (simulation case $A$ ). The dashed black lines are contour levels of $B / B_{0}$. Thin solid black lines show the magnetic lines that pass by the exit of the ionization chamber in this case. For comparison, the same lines in the symmetric configuration $15: 0.33: 0.33: 0.33$ (simulation case $O$ ) are shown as thin dotted white lines.

charged ions (' $i$ ') and electrons (' $e$ '). The plasma expansion is treated as quasineutral $\left(n_{i} \simeq n_{e} \equiv n\right)$, collisionless, and low- $\beta$ (i.e., negligible induced magnetic field effects). Ion temperature is assumed negligible compared to electron temperature, $T_{i} \ll T_{e}$, and electron inertia is neglected with respect to ion inertia, $m_{e} \ll m_{i}$. Furthermore, the electron population is simplified as a Maxwellian, isotropic, isothermal species, so that $T_{e}=$ const, with $T_{e}=p_{e} / n$ (although extensions to more complex electron thermodynamic models are straightforward ${ }^{39}$ ). Under these assumptions, the steady-state equations of continuity and momentum of each species read:

$$
\begin{aligned}
\nabla \cdot\left(n \boldsymbol{u}_{i}\right) & =0 ; \quad \nabla \cdot\left(n \boldsymbol{u}_{e}\right)=0, \\
m_{i}\left(\boldsymbol{u}_{i} \cdot \nabla\right) \boldsymbol{u}_{i} & =-e \nabla \phi+e \boldsymbol{u}_{i} \times \boldsymbol{B}, \\
0 & =-T_{e} \nabla \ln n+e \nabla \phi-e \boldsymbol{u}_{e} \times \boldsymbol{B} .
\end{aligned}
$$

where all symbols have the same conventional meaning as in Ref. ${ }^{36}$.

Besides the Cartesian vector basis $\left\{\mathbf{1}_{x}, \mathbf{1}_{y}, \mathbf{1}_{z}\right\}$, a local magnetic vector basis $\left\{\mathbf{1}_{\|}, \mathbf{1}_{\perp}, \mathbf{1}_{\times}\right\}$is defined with $\mathbf{1}_{\|}=$ $\boldsymbol{B} / B, \kappa \mathbf{1}_{\perp}=\left(\mathbf{1}_{\|} \cdot \nabla\right) \mathbf{1}_{\|}$, and $\mathbf{1}_{\times}=\mathbf{1}_{\|} \times \mathbf{1}_{\perp}$, where $\kappa$ is the curvature of the magnetic lines $\left(\kappa=\mathbf{1}_{\perp} \cdot \nabla \ln B\right.$ in a low- $\beta$ problem). The fluid velocity of each species is decomposed into its parallel component and a drift vector along $\mathbf{1}_{\perp}$ and $\mathbf{1}_{\times}$, here denoted with a hat, i.e.,

$$
\boldsymbol{u}_{i}=u_{\| i} \mathbf{1}_{\|}+\hat{\boldsymbol{u}}_{i} ; \quad \boldsymbol{u}_{e}=u_{\| e} \mathbf{1}_{\|}+\hat{\boldsymbol{u}}_{e} .
$$




\begin{tabular}{l|l||c|c|c|c} 
Case & Ampere-turn ratios & $F\left(R_{S}\right) / F_{0}$ & $\psi\left(R_{S}\right)(\mathrm{deg})$ & $\theta\left(R_{S}\right)(\mathrm{deg})$ & $\theta_{B}(\mathrm{deg})$ \\
\hline \hline$O$ & $15: 0.33: 0.33: 0.33$ & 1.44 & N/A & 0.00 & 0.00 \\
\hline$A$ & $15: 1: 0: 0$ & 1.44 & & 5.66 & 5.76 \\
$A^{\prime}$ & $15: 5: 0: 0$ & 1.34 & 0.00 & 11.06 & 11.24 \\
$\bar{A}$ & $15: 2:-0.5:-0.5$ & 1.44 & (triangle vertex) & 14.43 & 14.66 \\
$\bar{A}^{\prime}$ & $15: 10:-2.5:-2.5$ & 1.32 & & 30.96 & 31.29 \\
\hline$B$ & $15: 0.5: 0.5: 0$ & 1.44 & & 2.86 & 2.91 \\
$B^{\prime}$ & $15: 2.5: 2.5: 0$ & 1.34 & 60.00 & 5.61 & 5.70 \\
$\bar{B}$ & $15: 1: 1:-1$ & 1.44 & (triangle edge) & 11.92 & 12.10 \\
$\bar{B}^{\prime}$ & $15: 5: 5:-5$ & 1.33 & & 26.17 & 26.52 \\
\hline
\end{tabular}

TABLE I. List of magnetic configurations studied in this work. The ampere-turn ratios for each case are given as (solenoid):(coil 1):(coil 2):(coil 3). The last four columns present thrust vector control performance values from simulation results: normalized thrust force magnitude $F\left(R_{S}\right) / F_{0}$, thrust azimuth and polar angles $\psi\left(R_{S}\right)$ and $\theta\left(R_{S}\right)$, and polar angle of the central magnetic field line $\theta_{B}\left(R_{S}\right)$ for $R_{S}=4.5 R$ (early in the expansion).

Finally, both species are assumed to be fully magnetized, which requires that the dimensionless ion Larmor radius based on the sonic velocity be small,

$$
\varepsilon_{i}=\frac{\sqrt{m_{i} T_{e}}}{e B_{0} R} \ll 1 .
$$

Of course, this condition ensures that the dimensionless electron Larmor radius is also small, as $\varepsilon_{e}=$ $\sqrt{m_{e} T_{e}} /(e B R) \ll \varepsilon_{i}$. This condition is satisfied for high magnetic strengths and/or light propellants; as an example, the VASIMR in helicon source-only mode has $\varepsilon_{i} \simeq 0.1$ when operated with argon, and $\varepsilon_{i} \simeq 0.01$ when operated with hydrogen ${ }^{19}$. Assumption (5) allows the asymptotic expansion of Eqs. (1)-(3) around the zeroLarmor radius limit, where the motion of each species is mainly along the magnetic lines. Indeed, as the plasma expands supersonically in the divergent VMN, ions and electrons have a parallel velocity of the order of the sonic velocity, $u_{\| i} \sim u_{\| e} \sim c_{s}=\sqrt{T_{e} / m_{i}}$, while the drift velocities (sum of the diamagnetic and $\boldsymbol{E} \times \boldsymbol{B}$ drifts) scale as $\hat{u}_{i} \sim \hat{u}_{e} \sim T_{e} / e B R=\varepsilon_{i} c_{s}$. Thus, in the limit $\varepsilon_{i} \rightarrow 0, \hat{\boldsymbol{u}}_{i}$ and $\hat{\boldsymbol{u}}_{e}$ are negligible, and ion and electron streamlines coincide with magnetic lines. Note that, in this limit, the ion Mach number is defined simply as $M=u_{\| i} / c_{s}$.

To zeroth-order in $\varepsilon_{i}$ then, and using the identity

$$
\left(u_{\| i} \mathbf{1}_{\|} \cdot \nabla\right)\left(u_{\| i} \mathbf{1}_{\|}\right)=\frac{1}{2} \frac{\partial u_{\| i}^{2}}{\partial \mathbf{1}_{\|}} \mathbf{1}_{\|}+\kappa u_{\| i}^{2} \mathbf{1}_{\perp},
$$

the Eqs. (1) and the projection of Eqs. (2)-(3) along $\mathbf{1}_{\|}$ are integrated along the magnetic streamlines into:

$$
\begin{aligned}
n u_{\| i} / B & =G_{i}, \\
n u_{\| e} / B & =G_{e}, \\
\frac{1}{2} m_{i} u_{\| i}^{2}+e \phi & =H_{i}, \\
T_{e} \ln n-e \phi & =H_{e},
\end{aligned}
$$

where $G_{i}, G_{e}, H_{i}$ and $H_{e}$ are integration constants to be evaluated from the initial conditions on each magnetic line. The surfaces defined by the total plasma enthalpy
$H=H_{i}+H_{e}=$ const are termed $H$-tubes. From the conservation of $H_{i}$ and $H_{e}$ along magnetic lines it is evident that the vector $\mathbf{1}_{\|}$is contained in the tangent space at each point of these tubes.

Observe that the resulting zeroth-order model is purely algebraic, so that the plasma properties $u_{\| i}, u_{\| e}, \phi, n$ in the VMN can be obtained by solving Eqs. (7)-(10) in a line-by-line fashion, with a solution that depends only on the local value of $B$. This solution scheme has been implemented into an open-source code named FUMAGNO, after fully-magnetized magnetic nozzle. The model can be normalized with $R, m_{i}, e$, and $T_{e}$. Likewise, we may normalize the number density $n$ and the magnetic field $\boldsymbol{B}$ with their values at the origin, $n_{0}$ and $B_{0}$.

While $\hat{\boldsymbol{u}}_{i}, \hat{\boldsymbol{u}}_{e}$ are of order $O\left(\varepsilon_{i}\right)$, the magnetic force terms $e B \hat{\boldsymbol{u}}_{i}, e B \hat{\boldsymbol{u}}_{e}$ are of zeroth-order and scale as $\sim$ $m_{i} c_{s}^{2} / R$. Indeed, in a fully-magnetized plasma, these terms are responsible for most of the confinement and streamline deflection of each species, with the electric forces in the $\mathbf{1}_{\perp}$ and $\mathbf{1}_{\times}$directions playing only a minor role. These terms are also directly related to the generation of magnetic thrust. Using Eqs. (9) and (10), we may write the $\mathbf{1}_{\perp}$ and $\mathbf{1}_{\times}$components of Eqs. (2) and (3) as:

$$
\begin{aligned}
& e B \hat{\boldsymbol{u}}_{i}=\mathbf{1}_{\|} \times \nabla H_{i}+m_{i} \kappa u_{\| i}^{2} \mathbf{1}_{\times}, \\
& e B \hat{\boldsymbol{u}}_{e}=-\mathbf{1}_{\|} \times \nabla H_{e} .
\end{aligned}
$$

These equations can be combined to form an expression of the first-order electric drift current $\hat{\boldsymbol{\jmath}}=\operatorname{en}\left(\hat{\boldsymbol{u}}_{i}-\hat{\boldsymbol{u}}_{e}\right)$,

$$
\frac{\hat{\boldsymbol{\jmath}}}{e n c_{s}}=\varepsilon_{i}\left[\frac{\mathbf{1}_{\|} \times \nabla H\left(R / T_{e}\right)}{\left(B / B_{0}\right)}+\frac{\kappa R M^{2}}{\left(B / B_{0}\right)} \mathbf{1}_{\times}\right],
$$

where the first contribution is a confining force that prevents the perpendicularly-outward expansion of the plasma, and is always tangent to the $H$-tubes. The second contribution, in turn, is an expanding force that deflects ions according to the local magnetic curvature, and may not be tangent to them. Observe that the first contribution is always diamagnetic, as the direction of the induced magnetic field it creates is essentially opposite to the applied field and tends to lower the magnetization 
of the plume, whereas the second contribution is always paramagnetic. Observe also that the full first-order solution of the model consists not only of the $\hat{\boldsymbol{u}}_{i}$ and $\hat{\boldsymbol{u}}_{e}$ from Eqs. (11) and (12), but also of first-order terms of the plasma density, $n$, and the parallel ion and electron velocities, $u_{\| i}$ and $u_{\| e}$. These other terms must be computed by solving Eqs. (1)-(3) to order $\varepsilon_{i}$ and are not computed in the present work.

To close the model, a sufficient set of boundary conditions needs to be provided. This set should result from the coupling of the VMN/plasma model with an adequate plasma source model. In the present work, and for the purpose of illustrating the plasma expansion and the TVC capabilities of the VMN, the following representative conditions are chosen at the plane $z=0$ of Fig. 2, for $x^{2}+y^{2} \leq R^{2}$ :

$$
\begin{aligned}
u_{\| i} & =u_{\| e}=\sqrt{T_{e} / m_{i}}, \\
n & =n_{0} \exp \left(-a \frac{x^{2}+y^{2}}{R^{2}}\right), \\
\phi & =0,
\end{aligned}
$$

i.e., ions are initially sonic $(M=1)$, the plasma jet is initially current-free in the $\mathbf{1}_{\|}$direction, the density profile is Gaussian, and the initial plane is an isopotential surface. The value $a=3 \ln 10$ is selected so that the density profile decreases gradually into the surrounding vacuum, with $n=10^{-3} n_{0}$ initially at the plasma jet border.

\section{PLASMA EXPANSION CHARACTERISTICS}

The basic features of the expansion (e.g. magnitude of the self-consistent electric potential drop and ion acceleration) resemble those of the $2 \mathrm{D} \mathrm{MN}$ in the fully magnetized limit for comparable area expansion ratio ${ }^{36}$. The 3D plasma density response predicted by the model for the magnetic configuration $A$ in Table I (electric current ratios $15: 1: 0: 0$ ) is shown in Fig. 3. As it can be observed, the shape of the initial density profile is approximately propagated downstream by the $3 \mathrm{D}$ magnetic field, with the maximum following closely the VMN magnetic centerline. A sample of $H$-tubes is also shown in the Figure. These tubes, which are supported on initial plane concentric circles, stop being concentric in downstream $z=$ const cross-sections, and develop a small ellipticity. Density contour levels on these cross-sections do not exactly match the $H$-tubes, as a small difference in the ratio between the local value of $B$ and its value upstream exists that gives rise to a differential expansion between the $+y$ and $-y$ parts of the plasma plume.

Figure 4 displays the evolution of the self-consistent electric potential in a meridional section of the VMN for the same simulation. The isopotential curves are oriented according to the deflected magnetic field, following the local value of $B$. Observe that ion acceleration can be directly inferred from this graph; an ion Mach number $M \simeq 3$ has been reached at $z \simeq 12 R$.
The two contributions of Eq. 13 to the first-order ionelectron drift velocity difference, $\left(\hat{\boldsymbol{u}}_{i}-\hat{\boldsymbol{u}}_{e}\right) / c_{s}=\hat{\boldsymbol{j}} /\left(e n c_{s}\right)$, are shown in Fig. 5 for simulation $A$. Note that due to our choice of plasma conditions at $z=0$, there is $\nabla H_{i} \equiv 0$ in the plasma domain, so it is possible to identify the first contribution of Eq. 13 (diamagnetic) with $\hat{\boldsymbol{u}}_{e} / \varepsilon_{i}$ and the second (paramagnetic) with $\hat{\boldsymbol{u}}_{i} / \varepsilon_{i}$ (Eqs. (11) and (12)).

As the magnetic flux in the VMN is conserved, the magnetic field strength decays as $B / B_{0} \sim R_{V}^{-2}(z)$, where $R_{V}(z)$ is the characteristic radius of the plasma tube at axial position $z$. Hence, the contribution of plasma enthalpy to the drift velocities increases downstream roughly as $R_{V}(z)$. As depicted in the top left plot of Fig. 5, this contribution reaches a normalized value of $O\left(10^{2}\right)$ within the simulation domain. On the other hand, assuming that $\kappa \sim R_{V}^{-b}(z)$, with $b \in[0,1]$, the curvature contribution to the ion drift velocity grows faster than the former one as $R_{V}^{2-b}(z) M^{2}$. The direction of the total drift current $\hat{\boldsymbol{j}}$ is a crucial aspect of the expansion, on which the generation of magnetic thrust and the deflection of the thrust vector depend. Both the enthalpy and curvature contributions are larger at the periphery of the plasma, and have a dominant component along the $\mathbf{1}_{\times}$direction. Consistent with their dia- and paramagnetic nature, the two contributions have essentially opposite directions in most of the domain, as can be observed also in the right plots of Fig. 5, and therefore tend to partially cancel each other out. A net diamagnetic plasma is required for positive magnetic thrust generation ${ }^{24}$.

From the top right plot in Fig. 5 it is apparent that, while the contour levels of the plasma enthalpy contribution roughly agree with the magnetic streamtubes supported on circles at $z=0$, an asymmetry exists in the contribution magnitude between the $-y$ and $+y$ edges of the plasma cross-section. Quite differently, the curvature contribution shown in the bottom right plot of Fig. 5 does not follow the magnetic tubes; instead, it markedly depends on the local value of $\kappa$ and the local direction of the unit vector $\mathbf{1}_{\perp}$, which gives it an important $3 \mathrm{D}$ behavior. Indeed, as it can be inferred from the bottom left plot of Fig. 5, this contribution creates a drift ion velocity that essentially points in the $+\mathbf{1}_{x}$ direction between $z \simeq 1$ and 3 , where all magnetic lines of the meridional plane are curved toward the $-\mathbf{1}_{y}$ direction. Lastly, note that the two contributions to $\hat{\boldsymbol{j}} /\left(e n c_{s}\right)$ are $3 \mathrm{D}$ vectors which have in general an out-of-plane component in the right plots of Fig. 5, which is not depicted by the arrows of these plots. This out-of-plane component in the $\mathbf{1}_{z}$ direction is about $10 \%$ of the vector magnitude at $z=5 R$ in the periphery of the plasma plume.

\section{THRUST VECTOR CONTROL PERFORMANCE}

To analyze the TVC capabilities of the VMN it is necessary to define a few figures of merit. Firstly, the integral of the total plasma momentum flux over a spherical surface $S\left(R_{S}\right)$ of radius $R_{S}>R$ and center at the origin 

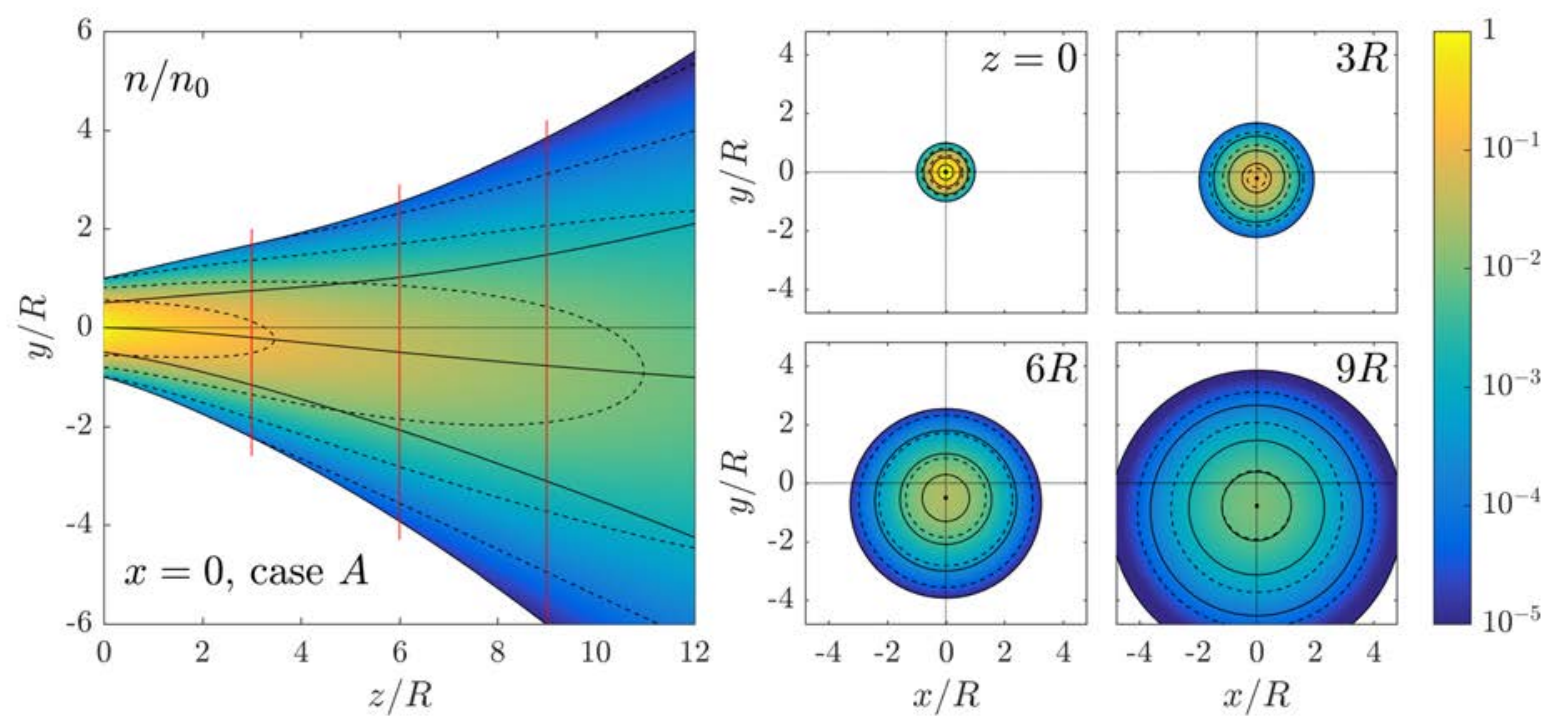

FIG. 3. Plasma density $n / n_{0}$ in the VMN, simulation case $A$ (electric current ratios $15: 1: 0: 0$ ). The plot on the left shows the plasma density on the meridional section of maximum deflection $(x=0)$. The small plots on the right show the plasma density on $z=$ const sections of the plume, whose $z$ position is indicated by the vertical red lines in the first plot. Solid black lines represent magnetic streamtubes supported on circles at $z=0$. Dashed black lines are plasma density contour lines.

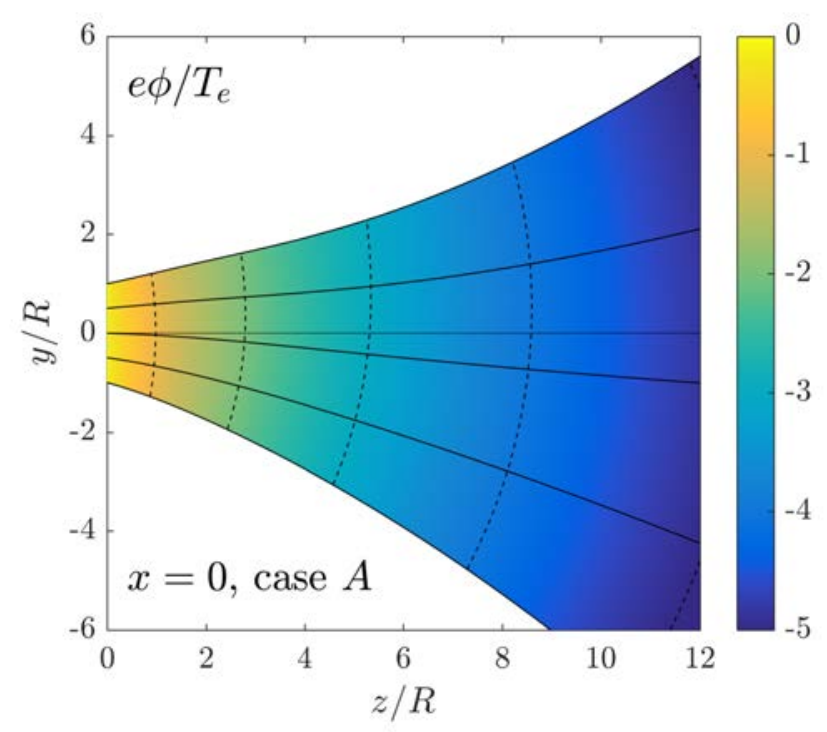

FIG. 4. Electric potential $e \phi / T_{e}$ on the meridional section of maximum deflection $(y=0)$, simulation case $A$ (electric current ratios $15: 1: 0: 0)$. Solid black lines are magnetic streamtubes; dashed black lines are isopotential lines.

defines the thrust force vector $\boldsymbol{F}\left(R_{S}\right)$ produced by the VMN up to that section, as a function of $R_{S}$. The components of this vector, i.e., $F_{x}\left(R_{S}\right), F_{y}\left(R_{S}\right)$ and $F_{z}\left(R_{S}\right)$, are given by:

$$
F_{k}\left(R_{S}\right)=\int_{S\left(R_{S}\right)} n\left(T_{e} \mathbf{1}_{k}+m_{i} u_{k i} u_{\| i} \mathbf{1}_{\|}\right) \cdot \mathrm{d} \boldsymbol{\sigma}
$$

where $k=x, y, z$. Then, the thrust azimuthal and polar angles, $\psi\left(R_{S}\right)$ and $\theta\left(R_{S}\right)$, are defined by

$$
\begin{aligned}
& \tan \psi\left(R_{S}\right)=-F_{x}\left(R_{S}\right) / F_{y}\left(R_{S}\right), \\
& \tan \theta\left(R_{S}\right)=\sqrt{F_{x}^{2}\left(R_{S}\right)+F_{y}^{2}\left(R_{S}\right)} / F_{z}\left(R_{S}\right) .
\end{aligned}
$$

Together with the thrust magnitude of $F\left(R_{S}\right)$ normalized with the thrust at the initial plane $z=0$, i.e. $F\left(R_{S}\right) / F_{0}$, the value of these angles is given in Table I for all the magnetic configuration cases defined in Section II. The value $R_{S}=4.5 R$ is chosen to study the TVC performance early in the expansion.

A series of observations can be made from these results: firstly, feeding an equal number of ampere-turns to each VMN coil indeed produces an axially-directed plasma plume and thrust vector which is identical to the equivalent $2 \mathrm{D}$ axisymmetric magnetic nozzle to all practical purposes.

Secondly, the VMN succeeds in effectively steering the thrust vector in any azimuthal direction when the electric current on the coils is varied. The polar deflection capability toward one of the vertices of the reference triangle of Fig. 1 (simulations of class $A$ ) is larger than toward its edges (simulations of class $B$ ), as expected.

Thirdly, as the overall solenoid/VMN current ratio is changed from 15:1 (unprimed simulations) to $15: 5$ (primed simulations), the greater authority of the VMN coils results in a stronger steering of the resulting magnetic field and hence a higher deflection angle $\theta$ of the thrust vector. In the limit of no solenoid current, the plasma jet would approach the limit deflection angle, which is equal to $\alpha$ for $A$-type simulations (15 deg in 

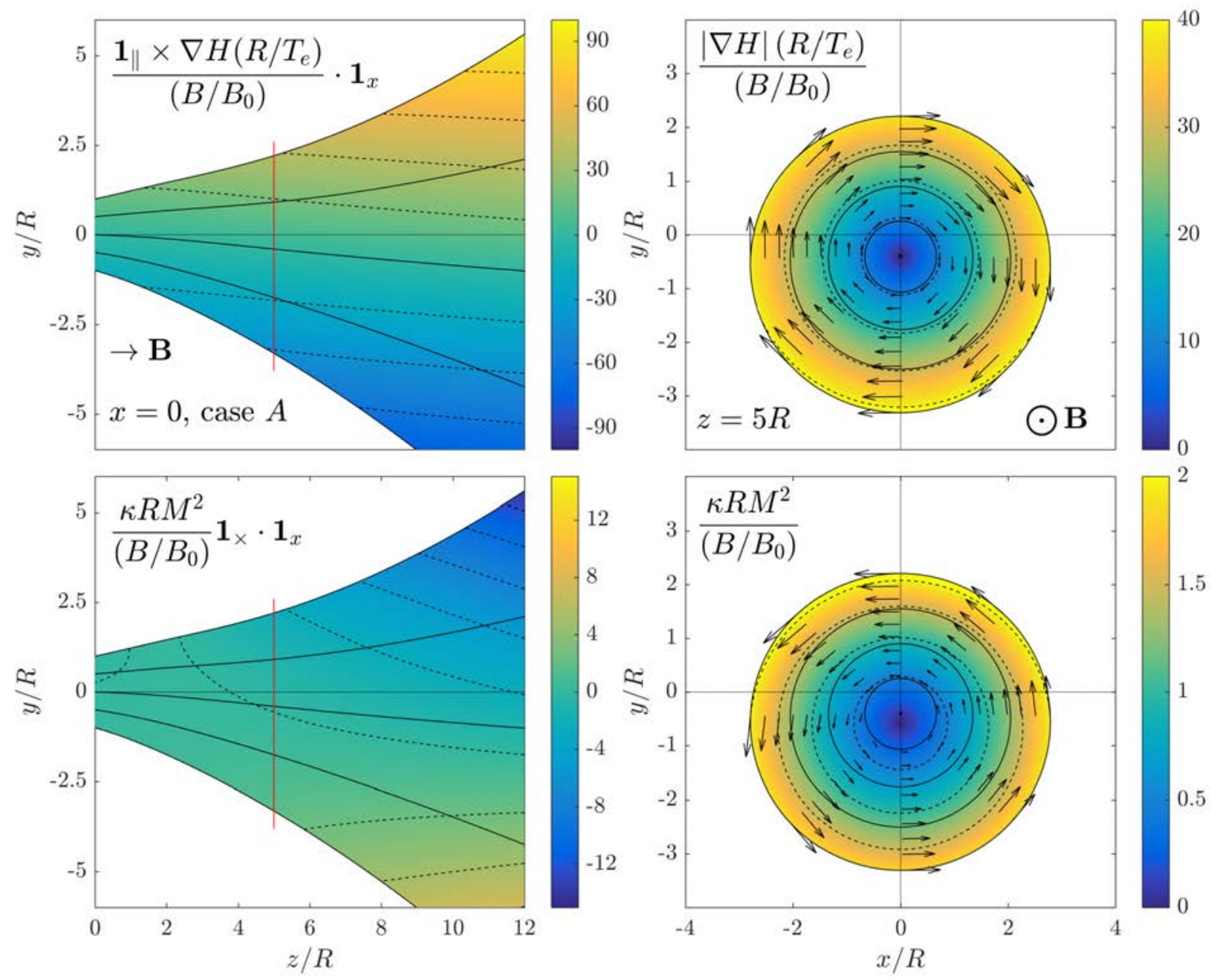

FIG. 5. First-order electric drift current $\hat{\boldsymbol{\jmath}} /($ en $)$ terms due to total plasma enthalpy gradient, $\nabla H$, (upper plots) and due to streamline curvature and ion inertia, $\kappa M^{2}$, (lower plots), as derived in Eq. 13. The left plots represent the meridional plane of maximum deflection $(x=0)$. As this plane is a symmetry plane, both vector contributions are parallel to $\pm \mathbf{1}_{x}$ in it. The plots to the right are the cross-sections of the plasma plume with the $z=5 R$ plane, indicated with red vertical lines in the plots to the left. The magnitude of each term is shown in the corresponding color map. Arrows show the direction and relative magnitude of each drift current contribution in the $z=$ const plane. In all plots, solid black lines represent magnetic streamtubes supported on circles at $z=0$. Dashed black lines are contour curves of the plotted quantities. The sign criterion for each contribution assumes the general direction of the $\boldsymbol{B}$ field as shown in the upper plots.

present cases), and $\arctan [\tan \alpha \sin (\pi / N)](\simeq 7.6 \mathrm{deg}$ in this example) for $B$-type cases. Naturally, this limit cannot be reached without substantially perturbing the magnetic topology inside the plasma source.

Fourthly, reversing the sign of one of the currents in the VMN coils allows reaching polar deflection angles well beyond those of the reference triangle and even beyond $\alpha$ (simulations with bar symbol), at the expense of a higher absolute value of the required current and therefore the power demand on the coils. Naturally, there is a limit to the maximum negative currents that can be used without significantly distorting the topology of the MN and affecting the expansion. This limit will depend on the values of $R_{L} / R, N$ and $\alpha$, and the details of the mag- netic configuration of the plasma source.

Fifthly, it is also noted that increasing the overall current in the VMN while keeping the solenoid current constant results in a longer, less divergent nozzle (the position of the the turning point of the outermost plasma streamlines is roughly $z_{L} \simeq 43 R$ for unprimed simulations and $z_{L} \simeq 65 R$ for primed ones). The lower area expansion ratio at $R_{S}=4.5 R$ for primed simulations results in a lower thrust magnitude $F / F_{0}$ at that distance. This illustrates one of the flexible aspects of general MNs, whose shape and strength can be adapted in-flight to suit the varying propulsive needs. Observe that to compare the generated thrust among MNs of different lengths, the tabulation would need to be done against constant area 
expansion ratio instead ${ }^{24}$. In general, longer nozzles result in lower divergence losses and therefore a more efficient plasma expansion, at the expense of a greater power and/or mass cost for the magnetic generator.

Sixthly, taking this into account, in simulations with the same overall solenoid/VMN current ratio, the total thrust magnitude $F$ produced by the VMN remains essentially constant in spite of the deflection, degrading only slightly for very high values of $\theta$ (simulations $\bar{A}^{\prime}$ and $\bar{B}^{\prime}$ ). This suggests that the VMN can operate in a wide angular deflection space without incurring in a noticeable performance deterioration.

Seventhly, it is apparent that the polar angle $\theta$ of the thrust force is lower than the polar angle of the central magnetic line of the VMN, $\theta_{B}$, also shown in Table I for comparison. This is due to two combined effects. On the one hand, the average magnetic line deflection is different from the deflection of the centerline. Secondly, as seen in Section III, all plasma properties depend on the relative drop of $B$ along each line. The magnetic strength $B$ does not decrease equally along each streamline, so that at $R_{S}=4.5 R$ the relative change of the plasma properties on each streamline is different; this causes a slight mismatch between the maximum of the plasma density on the spherical surface and the magnetic centerline.

Lastly, note that the power needed to operate the VMN is roughly $(1 / \cos \alpha)$ times larger than the corresponding single-coil MN with same mean radius and total coil mass. Thus, while increasing $\alpha$ extends the accessible angular space for deflection, it also increases the power budget of the thruster. Likewise, while breaking down the VMN into a larger number $N$ of lighter coils means more flexibility and a more uniform accessible deflection space in all directions (i.e., the reference polygon approaches a limit circumference as $N \rightarrow \infty$ ), it also increases the complexity of the device.

\section{ON PARTIALLY MAGNETIZED IONS}

In current MN-enabled thruster designs, the magnetic field strength ensures full electron magnetization in a large domain, but it is insufficient to keep the heavier ions magnetized far downstream. Expected values of the ion magnetization parameter $\varepsilon_{i}$ in propulsive applications range from $\varepsilon_{i} \simeq 0.01$ for light ions and strong magnetic fields (e.g. devices of the VASIMR-type running on hydrogen and superconductor coils) to $\varepsilon_{i} \simeq 1$ for heavy ions and mild/low magnetic field strengths. As shown in Ref. ${ }^{36}$, ion magnetization has little influence on thrust generation and ion trajectories in the near-region of a symmetric $\mathrm{MN}$; however, ion magnetization does affect plasma detachment in the far-region, and could also play a role on the lateral deflection capabilities of a VMN.

Therefore, it is worth commenting in more detail on the full magnetization assumption on which the VMN/plasma model presented in Section III hinges, the validity and limitations of the zeroth-order solution in $\varepsilon_{i}$ used in Sections IV and V, and the expected consequences of incomplete ion magnetization, i.e., when $\varepsilon_{i}$ is not necessarily small.

Firstly, it is observed that as the first-order drift current $\hat{\boldsymbol{j}}$ grows monotonically in magnitude along the plasma plume, the asymptotic expansion in $\varepsilon_{i}$ is not uniformly valid for all $z$. Consequent with this limitation, the zeroth-order solution can be regarded an adequate approximation of the plasma expansion only in a limited domain, up to about $z \sim 10 R$ for the example magnetic configuration of Section II with $\varepsilon_{i} \lesssim 0.1$. In other words, the zeroth-order model holds in the near-region plasma plume for mild values of $\varepsilon_{i}$. Beyond that, higherorder corrections become comparable to the zeroth-order terms.

Nevertheless, most of the plasma jet deflection takes place in the near region of the VMN, where the applied magnetic field experiences the largest reorientation, and thus in the domain where the zeroth-order approximation is still a reasonable one. Note that the near-region is also the region where ion Mach number $M$ is still small, which means that ion trajectories are still relatively easy to steer with a moderate force. This is indeed a desirable feature of the VMN, as early ion deflection allows operating at lower magnetic field strengths.

Secondly, it is possible to advance some of the expected implications of a partial ion magnetization in the VMN from previous works on axisymmetric $\mathrm{MNs}^{24,26}$. In an axisymmetric MN, when high-velocity ions demagnetize, their inertia makes them separate inward from the guiding magnetic lines. This mechanism is desirable and responsible for the so-called detachment process in the downstream region, which prevents the plasma from returning back to the thruster along the closed magnetic lines and limits its paramagnetic character, which cancels the produced magnetic thrust ${ }^{26}$. As a result of this separation, differential parallel electric currents appear in the plasma plume while complying with the global currentfree condition, and a perpendicular electric field develops in the plasma to prevent charge separation.

In a VMN with partially-magnetized ions, a similar behavior is expected: as the magnetic force on ions becomes insufficient to deflect them laterally to fully match the geometry of the guiding magnetic lines, ion separation occurs and their trajectories become more straight. This phenomenon is enhanced by the increasing ion inertia as the plasma expands, measured by the ion Mach number $M$. Consequently, the condition $\boldsymbol{u}_{i} \simeq u_{\| i} \mathbf{1}_{\|}$breaks down first as ions demagnetize, and the actual curvature of ion streamlines is less than $\kappa$. As a result, the first-order estimate of the paramagnetic ion drift velocity $\hat{\boldsymbol{u}}_{i}$ due to the magnetic curvature $\kappa$ shown in the bottom plots of Fig. 5 is an upper bound.

As long as electrons remain well magnetized, the ambipolar electric field in the plasma will supplement the magnetic force for the deflection of the ions, effectively steering the thrust vector. However, a lower deflection angle $\theta$ is expected in this case, as the maximum in the 
plasma density profile will shift farther away from the centerline of the VMN due to the separation of ions. Hence, the values obtained with the current model and portrayed in Table I should be regarded as the upper limit to deflection in the full magnetization limit. The detailed analysis of this separation would help determine the minimal value of the magnetic field strength required for the correct deflection of the plasma jet, if any.

Thirdly, it is pointed out that the first-order solution for the drift current $\hat{\boldsymbol{j}}$ derived in Eq. (13) may have a component out of the plume at the plasma-vacuum interface. Clearly, for a plasma expanding into vacuum, no normal current to this interface may exist. The introduction of this boundary condition on $\hat{\jmath}$ is expected to result in a thin current sheet on the plasma periphery similar to that of Ref. ${ }^{24}$. Electric currents along the two tangent directions of this last plasma streamtube would be required to satisfy current continuity. The analysis of this thin electric current layer is outside the scope of the present model.

To conclude, and in line with the comments above, a higher-order analysis of the present model, or altogether a 3D plasma model that does not rely on full ion magnetization assumption, is necessary to assess the deflection and plasma response as a function of the ion magnetization strength. It is reminded that the model presented here is strictly valid only in the limit of vanishing ion Larmor radius based on the sonic velocity, i.e. $\varepsilon_{i} \ll 1$, and in the limit of collisionless plasma, i.e., of collisional mean-free paths much larger than the characteristic macroscopic length of the problem, so it would not be applicable, in particular, to low-temperature collisional plasmas.

\section{CONCLUSIONS}

A 3D magnetic nozzle (or vector magnetic nozzle) has been presented that adds the capability of thrust vector control to the other advantages of magnetic nozzles for space electric propulsion applications. A possible implementation of the device has been proposed that uses several magnetic coils and no moving parts to achieve this effect. A fully-magnetized collisionless plasma model has been used to analyze the plasma expansion in the resulting 3D magnetic field, and to assess the thrust vector control capabilities of this setup.

Results of the plasma properties in the VMN evidence the capability of the applied magnetic field to guide the plasma expansion in the direction of the field. The analysis of the drift electric current shows that two dominant contributions exist at first order, namely a nearazimuthal diamagnetic electron current that creates a magnetic force to confine the total plasma enthalpy, and a paramagnetic ion current that creates an expanding magnetic force to deflect ions and match their zeroth-order trajectories to the magnetic lines of the VMN.

The analysis leads us to conclude that a VMN can be used to deflect a plasma jet in any direction within a designed conical angular space, in a contactless manner, and without mechanical moving parts, just by varying the electric current through several intertwined coils at the exit of the plasma source. In the fully-magnetized limit, the magnitude of the deflection depends dominantly on the VMN angle $\alpha$ and the electric current ratios on each coil. The perturbation to the internal magnetic topology is small in a wide parametric range, which allows decoupling the operation of the plasma source and the VMN. This form of magnetic deflection represents an interesting and flexible alternative to the existing TVC gimbaled platforms that are used in electric space propulsion assemblies and other magnetic deflection techniques, in particular in the case of plasma thrusters that already have a MN or a nearly-axial magnetic field. Lastly, further work must explore the operation of the VMN in the mild/low ion magnetization regime.

\section{ACKNOWLEDGMENTS}

The authors would like to thank Dr. Jesús Ramos from the Massachusetts Institute of Technology for the insightful discussions and comments during his stay as a visiting Chair of Excellence at Universidad Carlos III de Madrid. This work has been supported by the Spanish R\&D National Plan under grant number ESP2016-75887P.

${ }^{1}$ H. King, D. Schnelker, J. Ward, C. Dulgeroff, and R. Vahrenkamp, "Thrust vectoring systems," Tech. Rep. (1972).

${ }^{2}$ D. G. Fearn, "Ion thruster thrust vectoring requirements and techniques," in 27th International Electric Propulsion Conference, IEPC-01-115 (2001).

${ }^{3}$ J. Kugelberg, P. Bodin, S. Persson, and P. Rathsman, "Accommodating electric propulsion on smart-1," Acta Astronautica 55, 121-130 (2004).

${ }^{4}$ B. Wood, E. Gasparini, W. Buff, and A. Skulicz, "The development of a multi-purpose thruster orientation mechanism," in 14th European space mechanisms and tribology symposium (2011).

${ }^{5} \mathrm{~A}$. Wittmann, "Redundant system for satellite inclination control with electric thrusters," (2003), US Patent 6565043.

${ }^{6}$ A. Kural, N. Leveque, C. Welch, and P. Wolanski, "Design of an ion thruster movable grid thrust vectoring system," Acta Astronautica 55, 421-432 (2004).

${ }^{7}$ L. Garrigues, C. Boniface, G. Hagelaar, J. Boeuf, and O. Duchemin, "Performance modeling of a thrust vectoring device for Hall effect thrusters," Journal of propulsion and power 25, 1003-1012 (2009).

${ }^{8}$ W. Cox, C. Charles, R. Boswell, R. Laine, and M. Perren, "Magnetic ion beam deflection in the helicon double-layer thruster," Journal of propulsion and power 26, 1045-1052 (2010).

${ }^{9}$ S. Andersen, V. Jensen, P. Nielsen, and N. D'Angelo, "Continuous supersonic plasma wind tunnel," Phys. Fluids 12, 557-560 (1969).

${ }^{10} \mathrm{M}$. Merino and E. Ahedo, "Magnetic nozzles for space plasma thrusters," in Encyclopedia of Plasma Technology, Vol. 2, edited by J. L. Shohet (Taylor and Francis, 2016) pp. 1329-1351.

${ }^{11}$ O. Batishchev, "Minihelicon plasma thruster," IEEE Transaction on Plasma Science 37, 1563-1571 (2009).

${ }^{12} \mathrm{~K}$. Takahashi, A. Komuro, and A. Ando, "Effect of source diameter on helicon plasma thruster performance and its high power 
operation," Plasma Sources Science and Technology 24, 055004 (2015).

${ }^{13}$ J. C. Sercel, "Electron-cyclotron-resonance (ECR) plasma acceleration," in AIAA 19th Fluid Dynamics, Plasma Dynamics and Lasers Conference (1987).

${ }^{14}$ T. Lafleur, F. Cannat, J. Jarrige, P. Elias, and D. Packan, "Electron dynamics and ion acceleration in expanding-plasma thrusters," Plasma Sources Science and Technology 24, 065013 (2015).

${ }^{15}$ G. Krülle, M. Auweter-Kurtz, and A. Sasoh, "Technology and application aspects of applied field magnetoplasmadynamic propulsion," J. Propulsion and Power 14, 754-763 (1998).

${ }^{16}$ M. Zuin, R. Cavazzana, E. Martines, G. Serianni, V. Antoni, M. Bagatin, M. Andrenucci, F. Paganucci, and P. Rossetti, "Kink instability in applied-field magneto-plasma-dynamic thrusters," Physical review letters 92, 225003 (2004).

${ }^{17}$ C. Diaz, "The VASIMR rocket," Scientific American 283, 90-97 (2000).

${ }^{18}$ B. Longmier, E. Bering, M. Carter, L. Cassady, W. Chancery, F. Díaz, T. Glover, N. Hershkowitz, A. Ilin, G. McCaskill, et al., "Ambipolar ion acceleration in an expanding magnetic nozzle," Plasma Sources Science and Technology 20, 015007 (2011).

${ }^{19}$ C. Olsen, M. Ballenger, M. Carter, F. Chang Diaz, M. Giambusso, T. Glover, A. Ilin, J. Squire, B. Longmier, E. Bering, and P. Cloutier, "Investigation of plasma detachment from a magnetic nozzle in the plume of the VX-200 magnetoplasma thruster," Plasma Science, IEEE Transactions on 43, 252-268 (2015).

${ }^{20}$ K. Matyash, R. Schneider, A. Mutzke, O. Kalentev, F. Taccogna, N. Koch, and M. Schirra, "Kinetic simulations of spt and hemp thrusters including the near-field plume region," IEEE Transactions on Plasma Science 38, 2274-2280 (2010).

${ }^{21}$ D. Courtney and M. Martínez-Sánchez, "Diverging cusped-field Hall thruster," in 30th International Electric Propulsion Conference, Florence, Italy, IEPC-2007-39 (2007).

${ }^{22} \mathrm{D}$. Chubb, "Fully ionized quasi-one-dimensional magnetic nozzle flow," AIAA Journal 10, 113-114 (1972).

${ }^{23}$ R. Gerwin, G. Marklin, A. Sgro, and A. Glasser, "Characterization of plasma flow through magnetic nozzles," Tech. Rep. AFSOR AL-TR-89-092 (Los Alamos National Laboratory, 1990).

${ }^{24} \mathrm{E}$. Ahedo and M. Merino, "Two-dimensional supersonic plasma acceleration in a magnetic nozzle," Physics of Plasmas 17, 073501 (2010)

${ }^{25}$ E. Ahedo and M. Merino, "On plasma detachment in propulsive magnetic nozzles," Physics of Plasmas 18, 053504 (2011).
${ }^{26} \mathrm{M}$. Merino and E. Ahedo, "Plasma detachment in a propulsive magnetic nozzle via ion demagnetization," Plasma Sources Science and Technology 23, 032001 (2014).

${ }^{27} \mathrm{O}$. Okada and K. Kuriki, "The interaction between a plasma flow and a magnetic nozzle with strong Hall effect," ISAS report (1970).

${ }^{28}$ T. M. York, B. A. Jacoby, and P. Mikellides, "Plasma flow processes within magnetic nozzle configurations," Journal of Propulsion and Power 8, 1023-1030 (1992).

${ }^{29}$ M. Inutake, A. Ando, K. Hattori, H. Tobari, and T. Yagai, "Characteristics of a supersonic plasma flow in a magnetic nozzle," J. Plasma Fusion Res. 78, 1352-1360 (2002).

${ }^{30}$ C. Deline, R. Bengtson, B. Breizman, M. Tushentsov, J. Jones, D. Chavers, C. Dobson, and B. Schuettpelz, "Plume detachment from a magnetic nozzle," Physics of Plasmas 16, 033502 (2009).

${ }^{31}$ J. P. Sheehan, B. W. Longmier, E. A. Bering, C. S. Olsen, J. P. Squire, M. G. Ballenger, M. D. Carter, L. D. Cassady, F. R. C. Díaz, T. W. Glover, and A. V. Ilin, "Temperature gradients due to adiabatic plasma expansion in a magnetic nozzle," Plasma Sources Science and Technology 23, 045014 (2014).

$32 \mathrm{~J}$. Little and E. Choueiri, "Electron cooling in a magnetically expanding plasma," Physical Review Letters 117, 225003 (2016).

${ }^{33}$ K. Takahashi, A. Chiba, A. Komuro, and A. Ando, "Experimental identification of an azimuthal current in a magnetic nozzle of a radiofrequency plasma thruster," Plasma Sources Science and Technology 25, 055011 (2016)

${ }^{34}$ R. Hoyt, J. Scheuer, K. Schoenberg, R. Gerwin, R. Moses, and I. Henins, "Magnetic nozzle design for coaxial plasma accelerators," IEEE Transactions On Plasma Science 23, 481-494 (1995).

${ }^{35}$ K. Schoenberg, R. Gerwin, R. Moses, J. Scheuer, and H. Wagner, "Magnetohydrodynamic flow physics of magnetically nozzled plasma accelerators with applications to advanced manufacturing," Physics of Plasmas 5, 2090-2104 (1998).

${ }^{36} \mathrm{M}$. Merino and E. Ahedo, "Fully magnetized plasma flow in a magnetic nozzle," Physics of Plasmas 23, 023506 (2016).

${ }^{37}$ M. Merino and E. Ahedo, "Sistema sin partes móviles ni electrodos y procedimiento para vectorizar el empuje en motores espaciales de plasma," (2013), spanish Patent Office, Patent no. P201331790.

${ }^{38} \mathrm{M}$. Merino and E. Ahedo, "Towards thrust vector control with a 3D steerable magnetic nozzle," in $34^{\text {th }}$ International Electric Propulsion Conference, IEPC-2015-414 (Electric Rocket Propulsion Society, Fairview Park, OH, 2015).

${ }^{39} \mathrm{M}$. Merino and E. Ahedo, "Influence of electron and ion thermodynamics on the magnetic nozzle plasma expansion," IEEE Transactions on Plasma Science 43, 244-251 (2015). 